The Magnetic and Velocity Fields of Solar Active Regions

ASP Conference Series, Vol. 46,1993

Harold Zirin, Gwaxiang Ai, and Haimin Wang (eds.)

\title{
ACTIVE REGION EVOLUTION THROUGH COORDINATED OBSERVATIONS
}

\author{
N.MEIN ${ }^{1}$, P. MEIN ${ }^{1}$, B. SCHMIEDER ${ }^{1}$, O. ENGVOLD ${ }^{2}$, R. MOLOWNY ${ }^{2}$, \\ G.X. $\mathbf{A I}^{3}$ \\ ' Observatoire de Paris, Section de Meudon, F92195 Meudon Cedex \\ ${ }^{2}$ Institute of theoretical astrophysics, PO Box 1029, Blindern, N0315 \\ Oslo, Norway \\ ${ }^{3}$ Beijing Astronomical Observatory, Chinese Academy of Sciences, Beijing \\ 100080, P.R.China
}

\section{COORDINATED OBSERVATIONS}

On May 7, 1991, several ground-based instruments have been coordinated to observe AR 6615 (S10-W26), before and during the flight of the SERTS rocket experiment (Schmieder et al.,1992):

- Imaging spectroscopy (MSDP) at the VTT telescope of Tenerife (collaboration Paris-Meudon and KIS Freiburg). $H_{\alpha}$ and $N a D_{1}$ are observed simultaneously in 2D fields, with 9 and 11 wavelengths respectively (Mein, 1991).

- High resolution "white light" pictures (around $4686 \AA$ ) at the Swedish Vacuum Solar Telescope (SVST) of La Palma (Scharmer et al., 1985).

We present below some data from both instruments. Magnetic field maps are also available from the Huairou magnetograph (China), and from the GCT telescope (KIS/Tenerife).

\section{MSDP IMAGING SPECTROSCOPY}

As an example, the figure (I) shows the $H_{\alpha}$ intensity maps derived from the MSDP observations at 8:05 and 18:08 UT. Two arch systems are clearly seen in the middle part of the field. The magnetic maps from Huairou show that they cross magnetic neutral lines. The arches are darker at 8:05, but remain still visible at 18:08. Individual arches cannot be followed between both times.

The computation of dopplershifts from MSDP data show blueshifts in the parts pointing at disk center, and redshifts in the opposite direction. Preliminary analysis of the velocity field and the loop geometry are presented in this issue (Mein et al.). Material is flowing down in both legs of the arches, and the geometry accounts for the observed line-of-sight velocities.

\section{WHITE LIGHT HIGH RESOLUTION PICTURES (SVST)}

The observations consist of 50 minutes long time series, consisting of the sharpest images from consecutive intervals of 20 seconds. Pixel-to-pixel sensitivity variation over the CCD $(400 \times 240)$ have been removed as usual by "flat field" and dark current. 

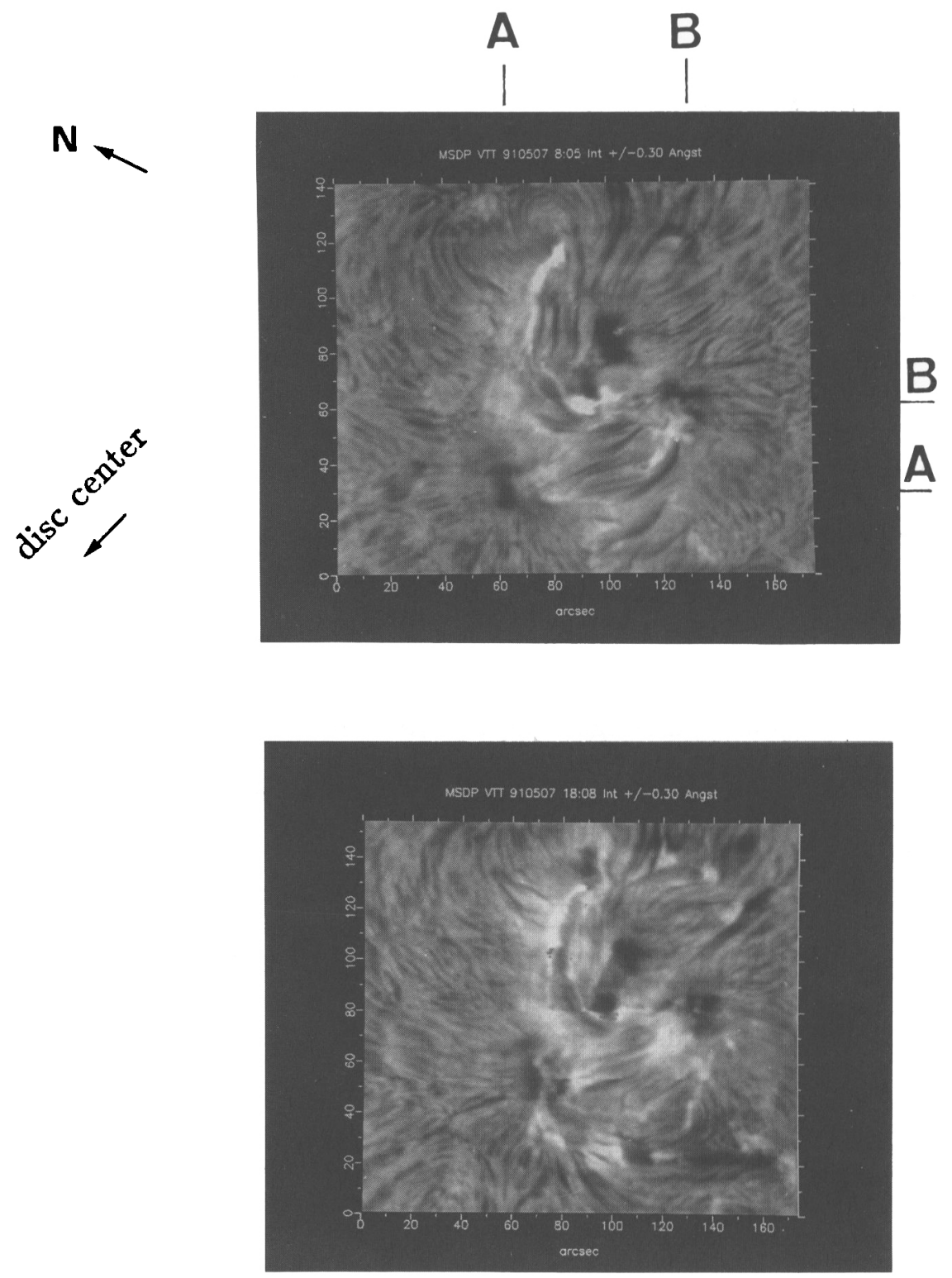

FIGURE I $H_{\alpha}$ intensity maps derived from MSDP data, at 8:05 UT (top) and 18:08 UT (bottom). 

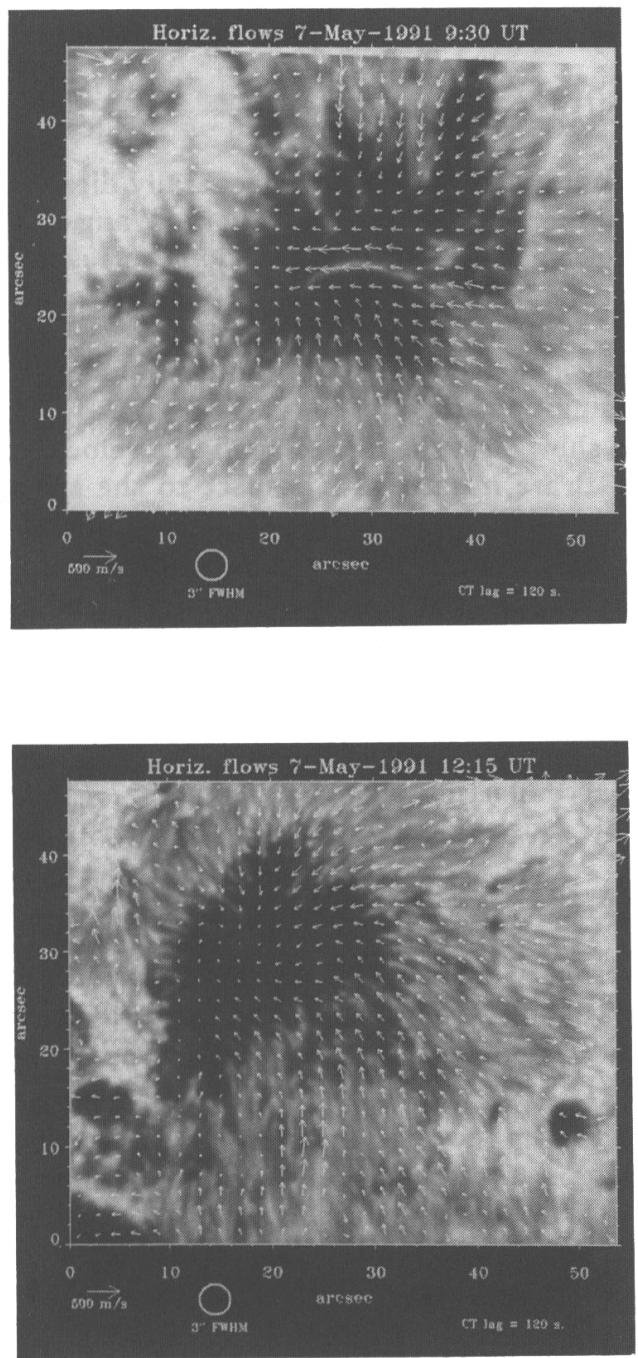

FIGURE II IIorizontal flow field from SVST data, around spots A (top) and B (bottom). 
The figure (II) shows "horizontal" flow velocities derived by the technique of Local Correlation Tracking (LCT)(November and Simon, 1988). The routines have been programmed by Molowny and Yi (1992). The fields of view are centered on two big spots, noted A and B on figure (I).

\section{PRELIMINARY RESULTS}

In spot regions, horizontal flows (fig. II) are generally directed inward in the inner half of the penumbrae, and outward elsewhere (Wang et al., 1992). However a different behaviour seems to occur in the regions corresponding to the feet of the $H_{\alpha}$ arches. Inward velocities are seen in the SW part of the field surrounding the spot $A$, even in the outer part of the penumbra. The same pattern can be seen in the NE part of field $B$. This might indicate that the magnetic loops of the arches are expanding upward.

The MSDP data show that the line-of-sight $H_{\alpha}$ velocities at the feet of the arches joining $\mathrm{A}$ and $\mathrm{B}$ are inward with respect to the spots, and that $\mathrm{NaD_{1 }}$ velocities are correlated with the same sign (Mein et al., this issue).

As a conclusion, all the velocity maps (white light, $\mathrm{H}_{\alpha}, \mathrm{NaD_{1 }}$ ) seem to indicate the same direction at the feet of the arches. This might be consistent with a model of material flowing down inside expanding magnetic loops (Mein et al., 1982, Tsiropoula et al., 1992).

Further investigations are necessary. In particular, the magnetic maps can be extrapolated in the upper layers of the solar atmosphere, and the dynamics can be analysed in connection with the geometry of magnetic lines. EUV observations will provide also valuable data on the transition region dynamics.

\section{ACKNOWLEDGMENTS}

We thank all the persons who helped us in this coordinated campaign, from Meudon and Canary Island Observatories, and from the german VTT and the swedish SVST. The MSDP images have been processed by the microdensitometer MAMA of CNRS (INSU).

\section{REFERENCES}

Mein P. 1991, Astron. Astrophys. 248, 669.

Mein P., Mein N. 1982, Solar Phys. 80, 161

Molowny R.H., Yi Z. 1992, LEST technical report 57, in press.

November L.J., Simon G.W. 1988, ApJ 333, 427.

Scharmer G.B., Brown D.S., Petterson L., Rehn J. 1985, Applied Optics 24, $N^{0}$ $16, \mathrm{p} 2558$.

Schmieder B., Mein N., Golub L., Davila J.M., Brosius J., Thomas R. 1992, SOHO Annapolis meeting, ESA publication (in press).

Tsiropoula G., Georgakilas A.A., Alissandrakis C.E., Mein P. 1992, Astron. Astrophys., in press.

Wang H., Zirin H. 1992, Solar Phys. 140, 41. 